\title{
An Experimental Comparison of Touch and Pen Gestures on a Vertical Display
}

\author{
Yusuke Niiro ${ }^{1,2}$, Marcelo Kallmann ${ }^{2}$, Ahmed Sabbir Arif ${ }^{1}$ \\ ${ }^{1}$ Human-Computer Interaction Group \\ ${ }^{2}$ Computer Graphics Lab \\ University of California, Merced \\ Merced, CA, USA \\ \{yniiro,mkallmann,asarif\}@ucmerced.edu
}

\begin{abstract}
We present results of a user study that compared touch and pen gestures on a vertical large display in terms of precision, duration, and difficulty. Results of the study revealed that touch gestures were significantly faster, while pen gestures were more precise. However, participants preferred using pen on the vertical display than touch. Results also revealed that performing gestures in the upper-middle area of the display was faster, more accurate, and easier compared to the other areas of the display.
\end{abstract}

\section{CCS CONCEPTS}

- Human-centered computing $\rightarrow$ gestural input; empirical studies in interaction design; touch screens.

\section{KEYWORDS}

vertical display; gesture; stylus; digital pen; interactive television.

\section{ACM Reference format:}

Yusuke Niiro, Marcelo Kallmann and Ahmed Sabbir Arif. 2019. An Experimental Comparison of Touch and Pen Gestures on a Vertical Display. In ACM International Symposium on Pervasive Displays (PerDis '19). Fune 12-14, 2019, Palermo, Italy. ACM, New York, NY, USA, 6 pages. https://doi.org/10.1145/1234567890

\section{INTRODUCTION}

With an increased availability and affordability, interactive large displays, such as interactive walls, tabletops, and televisions, are becoming a vital part of our daily life. Nowadays, we interact with large displays at airports, subways, commercial building, shopping malls, and even retail stores. Large displays are being used at work to create digital content and to acquire and visualize information, as well as at home for entertainment (e.g., big-screen television). The most common method of interaction with these devices are

Permission to make digital or hard copies of all or part of this work for personal or classroom use is granted without fee provided that copies are not made or distributed for profit or commercial advantage and that copies bear this notice and the full citation on the first page. Copyrights for components of this work owned by others than ACM must be honored. Abstracting with credit is permitted. To copy otherwise, or republish, to post on servers or to redistribute to lists, requires prior specific permission and/or a fee. Request permissions from Permissions@acm.org.

PerDis '19, fune 12-14, 2019, Palermo, Italy

(C) 2019 Association for Computing Machinery.

ACM ISBN 978-1-4503-6751-6/19/06...\$15.00

https://doi.org/10.1145/3321335.3324936 touch, digital pens or styli, and tangible objects [1,5]. However, a variety of commercial and academic solutions are available, such as remote controls, dials (e.g., [25]), and mid-air and whole-body gestures (e.g., [3,26]).

A considerable amount of research has focused on the design of novel interaction methods for large displays. Some have studied if large displays facilitate learning, collaboration, exploration, and scientific visualization (e.g., [2,7,10,13,16]). Some have also explored touch performance on horizontal displays. Many have studied the sociotechnical aspects of public large displays (e.g., $[17,22])$. Yet, to our knowledge, no prior research has compared touch and pen gestures on large displays in vertical orientation in terms of precision, duration, and difficulty. This paper attempts to bridge this gap through an empirical evaluation.

The rest of the paper is organized as follows. First, it provides an overview of existing research in the area. It then discusses the study design and motivation. It presents and discusses the results of the user study. Finally, it concludes with a reflection on future extension of the work.

\section{RELATED WORK}

Ardito et al. [1] conducted a literature review that revealed that most existing large interactive pubic displays use touch to "move, zoom, rotate, annotate objects, or provide other types of input", while some also use external devices, including tangible objects. A different survey reported similar results for interactive tabletops [5].

Rogers \& Lindley [18] studied the effects of screen orientation on groupwork. They found out that horizontal large displays facilitate collaboration, while collaborating on vertical displays is difficult and awkward, especially when performing tasks outside the display, such as taking notes or using a calculator.

Sasangohar et al. [19] evaluated mouse and touch input for a horizontal display using Fitts' reciprocal tapping task. In the study, touch yielded a higher throughput and a lower movement time than mouse. But error rates were much lower for the mouse than for touch. Pedersen \& Hornbæk [15] compared tap and drag on both vertical and horizontal displays. In their study, tapping was $5 \%$ faster on the vertical display, while dragging was $5 \%$ faster and more accurate on the horizontal display. Schick et al. [20] developed a method for enabling both touch and pointing on a very large 
vertical display. In a study, their method yielded a high accuracy rate. Besides, participants preferred using both touch and pointing rather than "only" touch. Nacenta et al. [14], in contrast, compared pantograph, telepointers, radar views, drag-and-drop, and laser beam interaction on a horizontal display. They reported significant effects of method on conflict, transfer, reaching, performance, and user preference. Arif \& Sylla [4] compared performance of touch and pen gestures on a tablet with both adults and children. Results revealed that pen gestures were significantly faster and more accurate than touch for adults. However, no effect was identified for children. Tu et al. [23] compared touch and pen gestures on a tablet in stationary and mobile settings. They reported that pen and index finger gestures are different in some features, i.e., size ratio, and pens are more effective in drawing precise and complex gestures.

Brandl et al. [6] compared three different input combinations for bimanual interaction on a horizontal display: touch-touch, pen-pen, and pen-touch. They found the pen-touch combination to be faster, more accurate, and the most preferred by the users than the other combinations. Kin et al. [11] compared direct touch, bimanual, and multi-finger interactions on a horizontal display for multi-target selection. Results revealed that direct touch with a single finger performed better than a mouse. Besides, bimanual interaction provided a smaller additional performance benefit. In another work, Forlines et al. [8] explored touch and mouse input for bimanual tasks and argued that "mouse input may be more appropriate for a single user working on tabletop tasks requiring only single-point interaction”. Matulic \& Norrie [12] designed novel pen and touch interaction methods for the editing and authoring of presentational documents on horizontal displays.

To our knowledge, no prior work has compared touch and pen gestures on large vertical displays in terms of precision, duration, and difficulty.

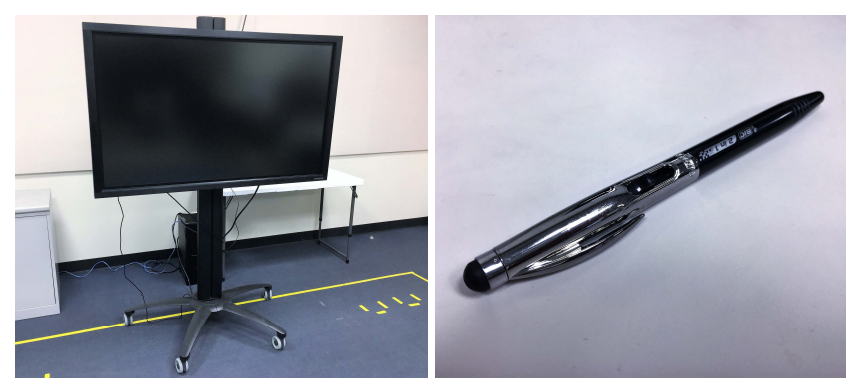

Figure 1. The interactive display (left) and the inactive pen or stylus (right) used during the user study.

\section{USER STUDY}

The purpose of this study was to investigate if different interaction modals, namely touch and pen, affects the precision, duration, and difficulty of gestures performed on a large vertical display.

\subsection{Apparatus}

We used a Christie Interactive $139.70 \mathrm{~cm}$ UHD LCD flat panel at $3840 \times 2160$ pixels. It detected touch using an integrated high- accuracy infrared touch technology [27]. It was mounted on a stand $93 \mathrm{~cm}$ above the floor in a vertical position for the users to view and reach the display "comfortably and without the adoption of extended postures" [21].

We used a BIC Tech 2 in 1 Retractable Ball Pen/Stylus, $17.8 \times$ $14.2 \times 6.8 \mathrm{~cm}$, tip diameter $7.5 \mathrm{~mm}, 18.14 \mathrm{~g}$, in the pen condition. This pen was selected after testing a number of commercial active and passive pens of different tip diameters $(1.6-8 \mathrm{~mm})$ and material (rubber and copper) on the display, where the selected tip yielded a higher accuracy rate and slid more smoothly on the surface (Figure 1).

\begin{tabular}{|c|c|c|c|}
\hline 1 & 2 & 3 & 4 \\
\hline 5 & 6 & 7 & 8 \\
\hline 9 & 10 & 11 & 12 \\
\hline
\end{tabular}

Figure 2. Overview of the 12 cells used to disply gestures.

\subsection{Cell and Gesture Sizes}

To investigate the effects of different display locations or zones on touch and pen gestures, we divided the display into 12 equal $310 \times 230$ pixels cells (Figure 2). This is comparable to a previous work that compared touch interactions between horizontal and vertical displays [15]. We intentionally left some inactive space around the edges (20 pixels on left and right and 15 pixels on the top and bottom) since the display's touch detection technology was unreliable in those areas.
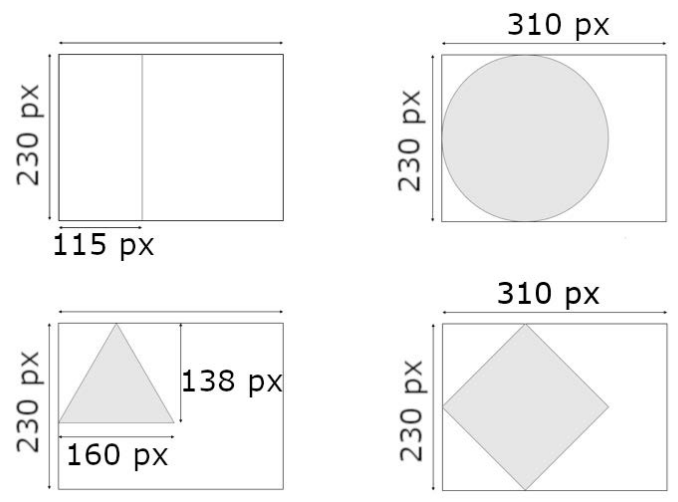

Figure 3. The gestures used in the study at their maximum size. In clockwise: line, circle, trangle, and square.

We used four different types of gestures in the study: line, circle, triangle, and square (Figure 3). Each of these gestures came in the following three sizes.

- $\quad$ Large: the largest gestures that can be freely rotated within a cell (i.e., they do not overlap with other cells when rotated). 
- Medium: gestures that are $3 / 4$ the size of large gestures.

- Small: gestures that are $1 / 2$ the size of large gestures. We picked this size since gestures smaller than this are often occluded under the fingertip.

\subsection{Application}

We developed a custom Web application using HTML5, CSS, PHP, and JavaScript for the study. It was loaded on a Google Chrome browser, running on Windows 10 OS on an Intel Core i7 machine. The application displayed one random gesture of a random size and angle on a random cell, and asked participants to trace it as fast and accurate as possible. The app used the \$1 recognizer [24] to process gestures. It calculated and logged all user performance directly. Figure 4 illustrates two participants interacting with the custom application via touch and pen.

\subsection{Metrics}

We recorded the following performance metrics in the study.

- Duration signifies the average time (in seconds) users took to perform a gesture. Timing started from the moment users touched the screen and ended when they lifted their fingers.

- Precision denotes how similar a trace was on average to the displayed gesture. Precision value ranges from 0 to 1 , where 1 suggests the presented and traced gestures were identical and 0 means the gestures were entirely different. This value was calculated using the $\$ 1$ recognizer [24]. There was almost no recognition error in the study since the system used only 4 gestures.

\subsection{Participants}

Twelve participants from the local university community, aged from 20 to 27 years $(M=22.33, S D=2.25)$, took part in the user study. Two of them were female and ten were male. One was left-handed, while the remaining 11 were right-handed. They had an average of 9.08 years $(\mathrm{SD}=1.93)$ experience with touch-based devices. Each of them received US $\$ 10$ for volunteering in the study.

\subsection{Design}

We used a within-subjects design for the user study. In summary, the design was:

12 participants $\times$

2 conditions (touch and pen, counterbalanced) $\times$

4 gestures (line, circle, triangle, rectangle, randomized) $\times$

3 sizes (large, medium, small, randomized, at a random angle) $\times$

12 cells $\times 2$ iterations

$=6,912$ gestures, in total, excluding practice.

\subsection{Procedure}

During the study, the custom application displayed one random gesture in a random size at a random angle on a random cell of the vertical display. They were then asked to trace it as fast and accurate as possible using either touch or pen in the corresponding condition in a counterbalanced order. Although the gestures, the gesture sizes and angles, and the display cells were randomized, the application displayed the same gesture set to all participants in order to eliminate a potential confounding variable.

The study was conducted in a quiet research lab. Upon arrival, participants were greeted and introduced to the interactive large display and the two interaction modes (touch and pen). We then explained the study procedure and collected their consents. They completed a demographics questionnaire that asked them about their age, gender, handedness, and experience with touchscreens. The main study started after that. We enabled participants to trace five gestures in a practice block using touch and pen before the respective conditions. There was a mandatory two minutes break between the conditions to reduce the effect of fatigue and stress. The study did not force participants to trace the same gesture in case of a mismatch (when participants entered a wrong gesture) or recognition error (when the application failed to recognize the gesture). The complete study was video recorded to observe user behavior.

Upon completion of all conditions, participants were asked to complete a questionnaire that asked them about their preferred interaction method and difficulty in performing the gestures in terms of physical and cognitive demand and stress.
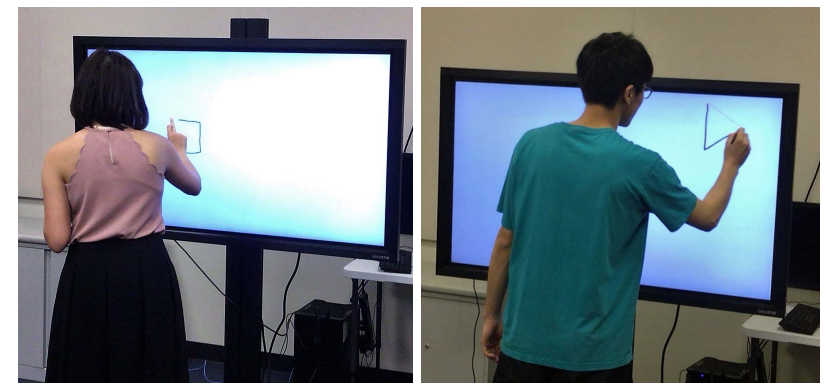

Figure 4. From left, users performing gestures on a vertical display using touch and pen.

\section{RESULTS}

A Shapiro-Wilk test failed to reject the null hypothesis $(p<.05)$ for all reported dependent variables that the sample came from a normally distributed population. A Mauchly's Test of Sphericity indicated that the assumption of sphericity had not been violated for any reported dependent variables $(p<.05)$. Hence, we used a repeated-measures ANOVA for all analysis.

\subsection{General Observations}

Almost all participants $(N=10,84 \%)$ used their right index fingers to perform the touch gestures. One participant (8\%) used the right middle finger and the reaming one left-handed participant $(8 \%)$ used the left index finger. Interestingly, almost all $(67 \%, N=8)$ participants occasionally switched to a different finger to perform some touch gestures, while the others $(33 \%, N=4)$ stuck to one finger throughout the study.

In the study, only $1.9 \%$ of all gestures were misrecognized by the application, primarily due to lifting fingers before finishing a gesture or for initiating a gesture in an inactive area. A researcher recorded these errors manually. 


\subsection{Precision}

An ANOVA identified a significant effect of condition $\left(F_{1,11}=14.61\right.$, $p=.002)$ on precision. Average precision rate for touch and pen were $0.92(\mathrm{SD}=0.23)$ and $0.95(\mathrm{SD}=0.09)$, respectively. An ANOVA also identified a significant effect of cell $\left(F_{1,11}=1.98, p=.03\right)$. A Tukey-Kramer Multiple-Comparison test revealed that performing gestures in cell 5 was the most error prone $(M=0.91)$, while in cell 3 was the most accurate $(M=0.95)$. Figure 5 shows average precision rate for all cells in both conditions.

\begin{tabular}{|l|l|l|l|}
\hline .928 & .924 & .940 & .934 \\
\hline .869 & .910 & .915 & .909 \\
\hline .908 & .908 & .926 & .933 \\
\hline
\end{tabular}

(a) Touch

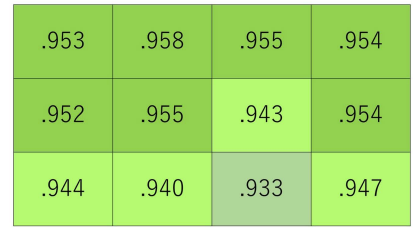

(b) Pen
Figure 5. Average precision rate for the twelve cells in both conditions.

Interestingly, there was no significant effect of gesture $\left(F_{1,11}=\right.$ $1.39, p=.26)$. Average precision rate for line, circle, triangle, and rectangle gestures were $0.93(\mathrm{SD}=0.21), 0.92(\mathrm{SD}=0.18), 0.94(\mathrm{SD}$ $=0.16)$, and $0.93(\mathrm{SD}=0.13)$, respectively. There was no significant effect of condition $\times$ cell $\left(F_{11,121}=1.82, p=.05\right)$ and condition $\times$ gesture $\left(F_{3,33}=1.18, p=.22\right)$ either.

\subsection{Duration}

An ANOVA identified a significant effect of condition $\left(F_{1,11}=6.02\right.$, $p=.03$ ) on duration. On average, touch and pen gestures took 1.63 $(\mathrm{SD}=1.09)$ and $2.01(\mathrm{SD}=1.36)$ seconds, respectively. An ANOVA also found a significant effect of cell $\left(F_{1,11}=11.45, p<.0001\right)$. A Tukey-Kramer Multiple-Comparison test revealed that drawing gestures in cells 9-12 took significantly more time than in cells 18 (2.0 vs. 1.7 seconds, respectively). Figure 6 illustrates average duration for all cells in both conditions.

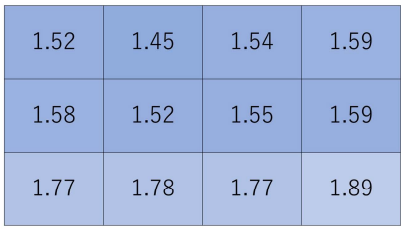

(a) Touch

\begin{tabular}{|c|c|c|c|}
\hline 1.92 & 1.89 & 1.80 & 1.96 \\
\hline 2.02 & 1.94 & 1.92 & 2.04 \\
\hline 2.21 & 2.16 & 2.16 & 2.18 \\
\hline
\end{tabular}

(b) Pen
Figure 6. Average gesture duration for the twelve cells in both conditions.

There was also a significant effect of gesture $\left(F_{1,11}=53.06, p<\right.$ .0001). On average, line, circle, triangle, and rectangle took 0.82 $(\mathrm{SD}=0.46), 2.33(\mathrm{SD}=1.42), 1.76(\mathrm{SD}=0.89)$, and $2.38(\mathrm{SD}=1.26)$ seconds, respectively. A Tukey-Kramer Multiple-Comparison test revealed that line was significantly faster than all other gestures (0.82 vs. 2.16 seconds, respectively).
An ANOVA failed to identify a significant effect of condition $\times$ cell $\left(F_{11,121}=1.17, p=.31\right)$, however, identified a significant effect of condition $\times$ gesture $\left(F_{3,33}=5.41, p=.003\right)$. A Tukey-Kramer Multiple-Comparison test revealed that performing circles, triangles, and rectangles were substantially slower with pen than with touch (2.38 vs. 1.93 seconds, respectively).

\section{USER FEEDBACK}

We used a Wilcoxon Signed-Rank and a Friedman test to analyze the post-study questionnaire data. The former is recommended for independent variables with two levels and the latter for more than two levels.

\subsection{Preference}

Participants were asked which interaction method (touch vs. pen) they preferred based on their experience in the user study. $75 \%$ participants $(N=9)$ preferred pen and the remaining 25\% $(N=3)$ preferred touch. There were not enough data to run a statistical test on this.

\subsection{Difficulty}

Participants were asked to rate the difficulty level of performing the gestures with touch and pen in terms of physical and cognitive demand or stress. A Wilcoxon Signed-Rank test failed to identify a significant effect of condition on difficulty $(p>.05)$. Median difficulty rating for both conditions were 2.0 on a 5 -point Likert scale, where rating 1-5 represented the least to the most difficult to perform gestures.

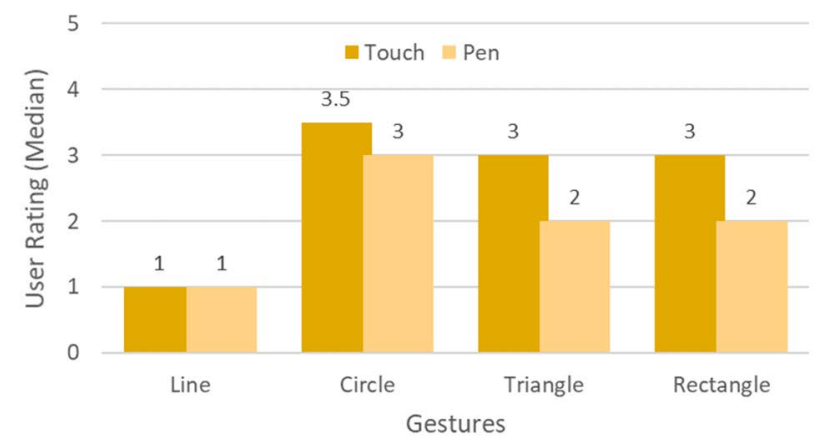

Figure 7. Median perceived difficulty in performing the four gestures on a 5-point Likert scale, where 1-5 represented the least to the most difficult to perform gestures.

A Friedman test identified a significant effect of gesture on difficulty $\left(\chi^{2}(3)=15.70, p<.0001\right)$. Figure 7 illustrates median rating of the four gestures on a 5-point Likert scale. In the figure, one can see that participants found the line gesture much easier to perform than the other gestures.

A Friedman test also identified a significant effect of cell on difficulty $\left(\chi^{2}(11)=9.54, p<.0001\right)$. Figure 8 illustrates median difficulty rating of touch and pen gestures in all cells on a 5-point Likert scale. 


\begin{tabular}{|c|c|c|c|}
\hline 2.5 & 2 & 2 & 2 \\
\hline 3 & 1.5 & 1 & 2 \\
\hline 4.5 & 3.5 & 3 & 4 \\
\hline
\end{tabular}

(a) Touch

\begin{tabular}{|l|l|l|l|}
\hline 2 & 2 & 1 & 1 \\
\hline 2 & 1 & 1 & 2 \\
\hline 4 & 3 & 3 & 3.5 \\
\hline
\end{tabular}

(b) Pen
Figure 8. Median difficulty rating of touch and pen gestures in all cells on a 5-point Likert scale, where 1-5 represented the least to the most difficult cells.

\section{DISCUSSION}

Results revealed that interaction method had a significant effect on precision. Evidently, gestures performed with the pen were $32 \%$ more precise than touch (Figure 5). Participants also recognized this, reflected in their response to the post-study questionnaire, where $75 \%$ of them wanted to use pen to interact with large displays. This result conforms to the findings of a previous user study [4], where gestures drawn with a pen on a tablet were significantly more precise than with touch. However, in that study, participants preferred using touch than pen. This could be due to the use of a different form-factor (tablet vs. large display) or the "novelty effect" [9:172] since touch was still a novel mode of interaction when that study was conducted in 2013. There was also a significant effect of cell. In Figure 5, one can see that the gestures performed on the upper two rows of the display were more precise in both touch and pen conditions than the bottom row. This is likely due to the fact that participants had to lean forward or lean down to reach the lower part [21]. Participant height may have had an impact on this. However, a larger sample is needed to explore this possibility. Interestingly, there was no significant effect of gesture on precision, which suggests that participants did not face any major difficulties in performing the gestures.

Results revealed that performing the gestures with touch was significantly faster. On average, touch gestures were $19 \%$ faster than pen gestures. This contradicts the findings of a prior study [4], where gestures performed with a pen on a tablet computer were significantly faster than with touch. Again, this may be due to the different form-factor (tablet vs. large display). There was also a significant effect of cell. In Figure 6, one can see that the middle cells of the upper two rows were much faster than other cells of the display. This conforms to the findings of a previous study [15] that also reported similar results for touch interaction on a large vertical display. User response to the post-study questionnaire suggests that participants also recognized this effect since they rated the middle cells of the upper two rows as less difficult than the other areas of the display (see Figure 8). Therefore, it may be prudent for interface designers to restrict important interactive items within this area.

As expected, there was also a significant effect of gesture on duration. The simplest gesture "line" was $62 \%$ faster than the other three gestures. Interestingly, performing the other gestures was $19 \%$ faster with touch than with pen $(p<.05)$. Participant responses to post-study questionnaire also reflected this, where line was rated as the least difficult gesture to perform (Figure 7).

\subsection{Recommendations}

Based on the findings of this work, we make the following design and development recommendations for large vertical displays.

- $\quad$ Map simpler gestures to the most frequent tasks (i.e., creating a folder, opening a new window, etc.), to increase the speed and accuracy of the system and to reduce the (perceived) cognitive and physical load.

- Provide the support for both touch and pen and avoid the "one design fits all" strategy for vertical displays, when possible. It is clear from the results that touch is effective for "interaction" tasks, where precision is not essential (e.g., drag and drop). Pen, on the other hand, is more effective for "input" tasks, where precision is desired (e.g., handwriting and sketching).

- Use different areas (cells) of the screen to display different interactive elements targeted at touch and pen. Specifically, place touch elements in areas whether they yielded the best performance and vice versa. But, we recommend placing the most important interactive elements in the areas where both touch and pen yielded the best performance so that those are usable and effective with either modality.

\section{CONCLUSION}

We compared touch and pen gestures on a vertical large display in terms of precision, duration, and difficulty. Results revealed that touch gestures were substantially faster, while pen gestures were more precise. Besides, participants preferred using pen on the vertical display than touch. Results also revealed that performing gestures in the middle of the upper two rows were not only faster and more accurate but also the least difficult compared to the other areas of the display.

We hope that these findings will assist designers in designing more effective interactive systems for large displays by accounting for the "difficult areas" of the display. This work must also encourage researchers and practitioners to explore and provide the support for both touch and pen-based interactions on vertical displays.

\section{FUTURE WORK}

In the future, we will compare the performance of touch and pen gestures on a horizontal display. We will also explore the effects of user height on performance and preference. In addition, we will extend the work to multi-touch, bimanual, and collaborative interactions where multiple users are working on a large display simultaneously.

\section{ACKNOWLEDGEMENTS}

We thank the participants of this user study. This work has been supported in part by the UC Merced Academic Senate Faculty Research Grants. 


\section{REFERENCES}

[1] Carmelo Ardito, Paolo Buono, Maria Francesca Costabile, and Giuseppe Desolda. 2015. Interaction with large displays: A survey. ACM Comput. Surv 47, 3 (February 2015), 1-38. DOI:https://doi.org/10.1145/2682623

[2] Ahmed Sabbir Arif, Roozbeh Manshaei, Sean Delong, Brien East, Matthew Kyan, and Ali Mazalek. 2016. Sparse Tangibles: collaborative exploration of gene networks using active tangibles and interactive tabletops. In Proceedings of the TEI '16: Tenth International Conference on Tangible, Embedded, and Embodied Interaction - TEI '16, 287-295. DOI:https://doi.org/10.1145/2839462.2839500

[3] Ahmed Sabbir Arif, W Stuerzlinger, and A Gordynski. 2014. Error behaviours in an unreliable in-air gesture recognizer. In CHI '14 Extended Abstracts on Human Factors in Computing Systems - CHI EA '14, 1603-1608. DOI:https://doi.org/10.1145/2559206.2581188

[4] Ahmed Sabbir Arif and Cristina Sylla. 2013. A comparative evaluation of touch and pen gestures for adult and child users. In Proceedings of the 12th International Conference on Interaction Design and Children - IDC '13, 392395. DOI:https://doi.org/10.1145/2485760.2485804

[5] Andrea Bellucci, Alessio Malizia, and Ignacio Aedo. 2014. Light on horizonta interactive surfaces: Input space for tabletop computing. ACM Comput. Surv 46, 3 (January 2014), 1-42. DOI:https://doi.org/10.1145/2500467

[6] Peter Brandl, Clifton Forlines, Daniel Wigdor, Michael Haller, and Chia Shen 2008. Combining and measuring the benefits of bimanual pen and directtouch interaction on horizontal interfaces. In Proceedings of the working conference on Advanced visual interfaces - AVI '08, 154 DOI:https://doi.org/10.1145/1385569.1385595

[7] Rebecca Fiebrink, Dan Morris, and Meredith Ringel Morris. 2009. Dynamic mapping of physical controls for tabletop groupware. In Proceedings of the 27 th international conference on Human factors in computing systems - CHI '09, 471. DOI:https://doi.org/10.1145/1518701.1518778

[8] Clifton Forlines, Daniel Wigdor, Chia Shen, and Ravin Balakrishnan. 2007. Direct-touch vs. mouse input for tabletop displays. In Proceedings of the SIGCHI conference on Human factors in computing systems - CHI '07, 647. DOI:https://doi.org/10.1145/1240624.1240726

[9] Frederick J. Gravetter and Lori-Ann B Forzano. 2015. Research Methods for the Behavioral Sciences (5th ed.). Cengage, Stamford, CT, USA

[10] Tobias Isenberg, Uta Hinrichs, and Sheelagh Carpendale. 2009. Studying direct-touch interaction for 2D flow visualization. In Collaborative Visualization on Interactive Surfaces - CoVIS' 09, 4 pages. Retrieved from https://www.lri.fr/ isenberg/publications/papers/Isenberg_2010_CoVis.pdf\#p age $=27$

[11] Kenrick Kin, Tony Derose, Maneesh Agrawala, and Tony Derose. 2009. Determining the benefits of direct-touch, bimanual, and multifinger input on a multitouch workstation. In Proceedings of the Graphics Interface Conference (GI'09), 119-124. Retrieved from http://portal.acm.org/citation.cfm?id=1555880.1555910

[12] Fabrice Matulic and Moira C. Norrie. 2013. Pen and touch gestural environment for document editing on interactive tabletops. In Proceedings of the 2013 ACM international conference on Interactive tabletops and surfaces ITS '13, 41-50. DOI:https://doi.org/10.1145/2512349.2512802

[13] Meghna Mehta, Ahmed Sabbir Arif, Apurva Gupta, Sean DeLong, Roozbeh Manshaei, Graceline Williams, Manasvi Lalwani, Sanjay Chandrasekharan, and Ali Mazalek. 2016. Active Pathways: Using Active Tangibles and Interactive Tabletops for Collaborative Modeling in Systems Biology. Proc. 2016 ACM Interact. Surfaces Spaces (2016), 129-138.
DOI:https://doi.org/10.1145/2992154.2992176

[14] Miguel A Nacenta, David Pinelle, Dane Stuckel, and Carl Gutwin. 2007. The effects of interaction technique on coordination in tabletop groupware. In Proceedings of Graphics Interface 2007 on - GI '07, 191. DOI:https://doi.org/10.1145/1268517.1268550

[15] Esben Warming Pedersen and Kasper Hornbæk. 2012. An experimenta comparison of touch interaction on vertical and horizontal surfaces. In Proceedings of the 7th Nordic Conference on Human-Computer Interaction Making Sense Through Design - NordiCHI '12, 370. DOI:https://doi.org/10.1145/2399016.2399074

[16] Peter Peltonen, Antti Salovaara, Giulio Jacucci, Tommi Ilmonen, Carmelo Ardito, Petri Saarikko, and Vikram Batra. 2007. Extending large-scale event participation with user-created mobile media on a public display. In Proceedings of the 6th international conference on Mobile and ubiquitous multimedia - MUM '07, 131-138. DOI:https://doi.org/10.1145/1329469.1329487

[17] Yvonne Rogers and Harry Brignull. 2002. Subtle ice-breaking: Encouraging socializing and interaction around a large public display. DOI:https://doi.org/10.2169/internalmedicine.43.527

[18] Yvonne Rogers and Siân Lindley. 2004. Collaborating around vertical and horizontal large interactive displays: which way is best? Interact. Comput. 16 , 6 (December 2004), 1133-1152. DOI:https://doi.org/10.1016/j.intcom.2004.07.008

[19] Farzan Sasangohar, I. Scott MacKenzie, and Stacey D Scott. 2009. Evaluation of mouse and touch input for a tabletop display using Fitts' reciprocal tapping task. Proc. Hum. Factors Ergon. Soc. Annu. Meet. 53, 12 (October 2009), 839-843. DOI:https://doi.org/10.1177/154193120905301216

[20] Alexander Schick, Florian van de Camp, Joris Ijsselmuiden, and Rainer Stiefelhagen. 2009. Extending touch: Towards interaction with large-scale surfaces. In Proceedings of the ACM International Conference on Interactive $\begin{array}{lllll}\text { Tabletops and Surfaces - ITS O9, } & 117 .\end{array}$ DOI:https://doi.org/10.1145/1731903.1731927

[21] Melanie Swann. 2006. Ergonomics of touch screens. Surrey, UK. Retrieved from https://www.ergonomic.solutions/wpcontent/uploads/2015/04/Touch_Screen_Ergonomics_06.pdf

[22] Martin Tomitsch, Christopher Ackad, Oliver Dawson, Luke Hespanhol, and Judy Kay. 2014. Who cares about the Content? An analysis of playful behaviour at a public display. In Proceedings of The International Symposium on Pervasive Displays - PerDis '14, 160-165. DOI:https://doi.org/10.1145/2611009.2611016

[23] Huawei Tu, Xiangshi Ren, and Shumin Zhai. 2015. Differences and similarities between finger and pen stroke gestures on stationary and mobile devices. ACM Trans. Comput. Interact. 22, 5 (August 2015), 1-39. DOI:https://doi.org/10.1145/2797138

[24] Jacob O Wobbrock, Andrew D Wilson, and Yang Li. 2007. Gestures without libraries, toolkits or training: A $\$ 1$ recognizer for user interface prototypes. In Proceedings of the 20th annual ACM symposium on User interface software and technology - UIST '07, 159. DOI:https://doi.org/10.1145/1294211.1294238

[25] Surface Dial - Microsoft Store. Retrieved February 3, 2019 from https://www.microsoft.com/en-us/p/surface-dial/925r551sktgn

[26] Kinect - Windows App Development. Retrieved February 3, 2019 from https://developer.microsoft.com/en-us/windows/kinect

27] Christie FHQ552-T 55" UHD Interactive LCD Flat Panel. Christie - Audio Visual Solutions Retrieved February 7, 2019 from https://www.christiedigital.com/en-us/digital-signage/products/LCD-flatpanels/christie-fhq552-t 